\title{
NMR relaxation studies of counter ion nuclei in solution
}

Nuwanthika Kumarage, and David Tierney

Department of Chemistry and Biochemistry, Miami University, Oxford, Ohio 45056

\section{Introduction}

Non covalent interaction between counter ion nuclei and metal center can affect the reactivity and chemical structure of the metal complex in solution. ${ }^{1}$ Many metal complexes accompanied counter ion to counterbalance its electric charge. However, nature of the counter ion and its effect on properties such as geometry, vibrations, nuclear spin content, and electronic spin magnitude has yet to be investigated. ${ }^{2}$ Previous NMR studies on unsubstituted $\left[\mathrm{Co}(\mathrm{Tpm})_{2}\right]^{+2}$ with $\mathrm{BF}_{4}^{-}$or $\mathrm{PF}_{6}^{-}$counter ions has shown that $\mathrm{BF}_{4}{ }^{-}$ counter ions are strongly associated with the metal center. ${ }^{3}$ In this project, Series of substituted cobalt (II) bis-trispyrazolylmethane $\left([\mathrm{Co}(\mathrm{Tpm}) 2]^{+2}\right)$ complexes was studied to understand the effect of counter ion on NMR relaxation, geometry and the spin cross over. Here we report temperature dependent relaxation rates of counter ion nuclei and distance between counter ion nuclei $\left(\mathrm{BF}_{4}{ }^{-}\right.$and $\left.\mathrm{PF}_{6}{ }^{-}\right)$ and the metal center

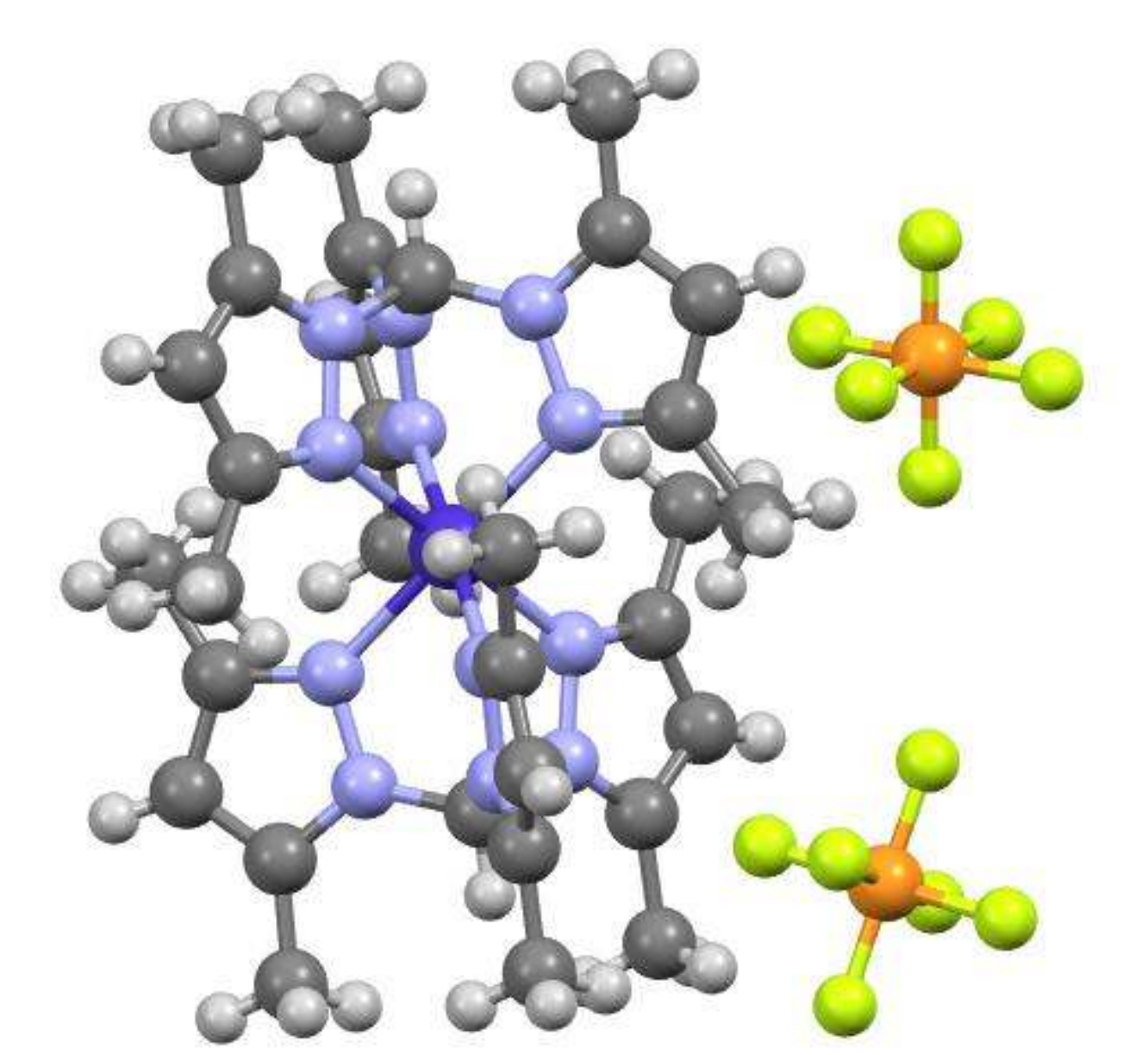

$\left[\mathrm{Co}\left(\mathrm{Tpm}^{3,5 \mathrm{me}}\right)_{2}\right]\left[\mathrm{PF}_{6}\right]$

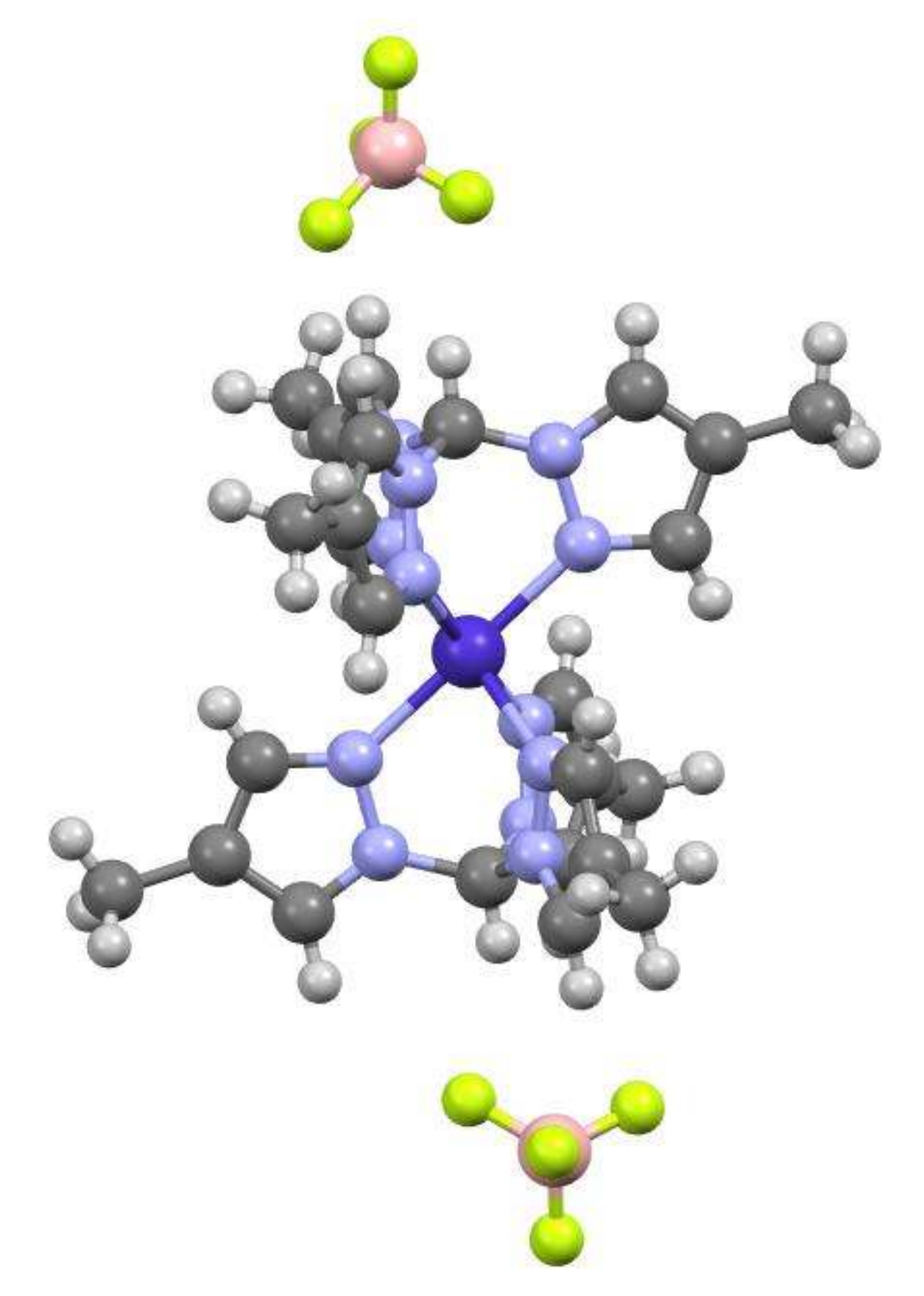

$\left[\mathrm{Co}\left(\mathrm{Tpm}^{4 \mathrm{me}}\right)_{2}\right]\left[\mathrm{BF}_{4}\right]$

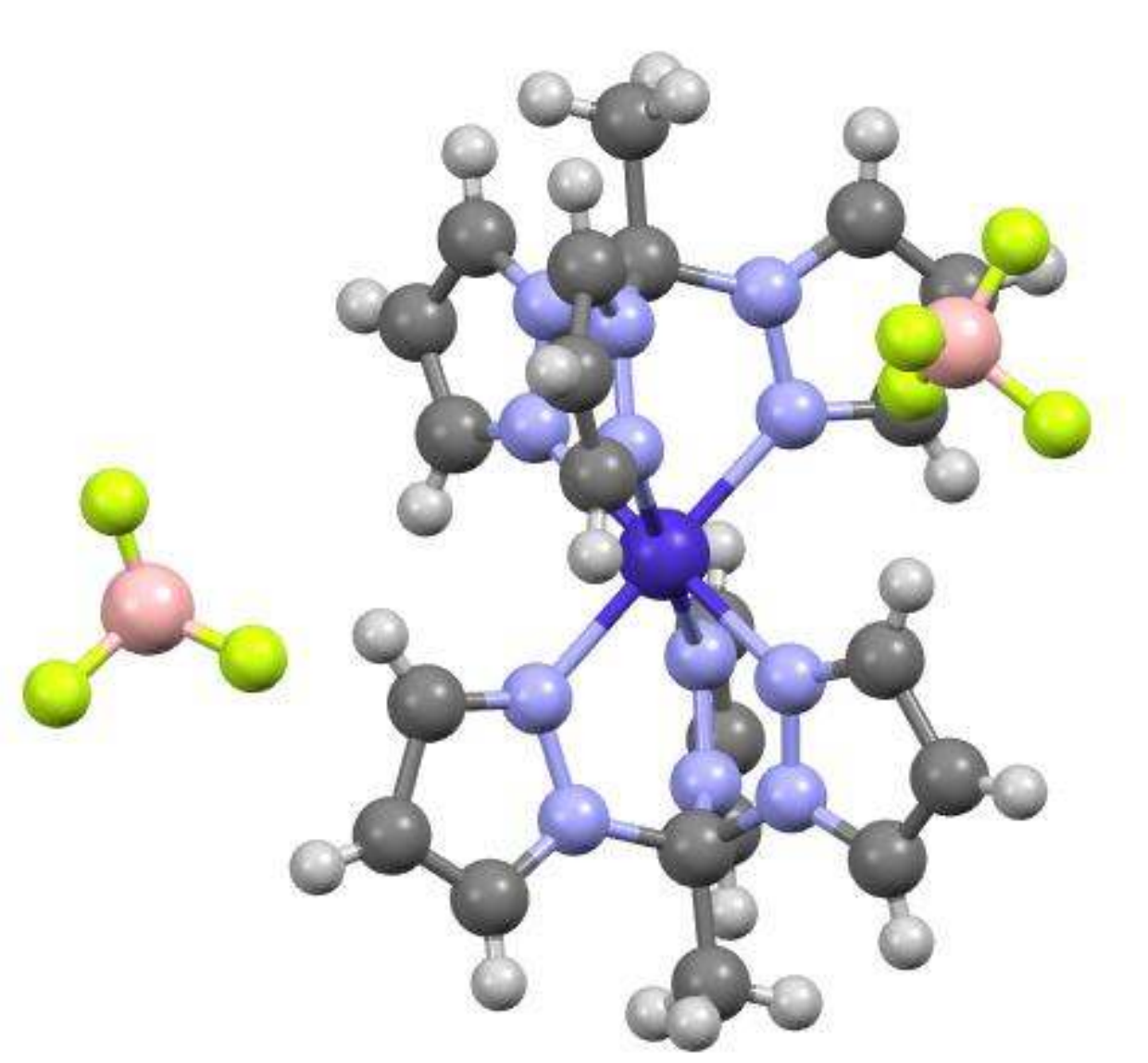

$\left[\mathrm{Co}(\mathrm{Tpe})_{2}\right]\left[\mathrm{BF}_{4}\right]$

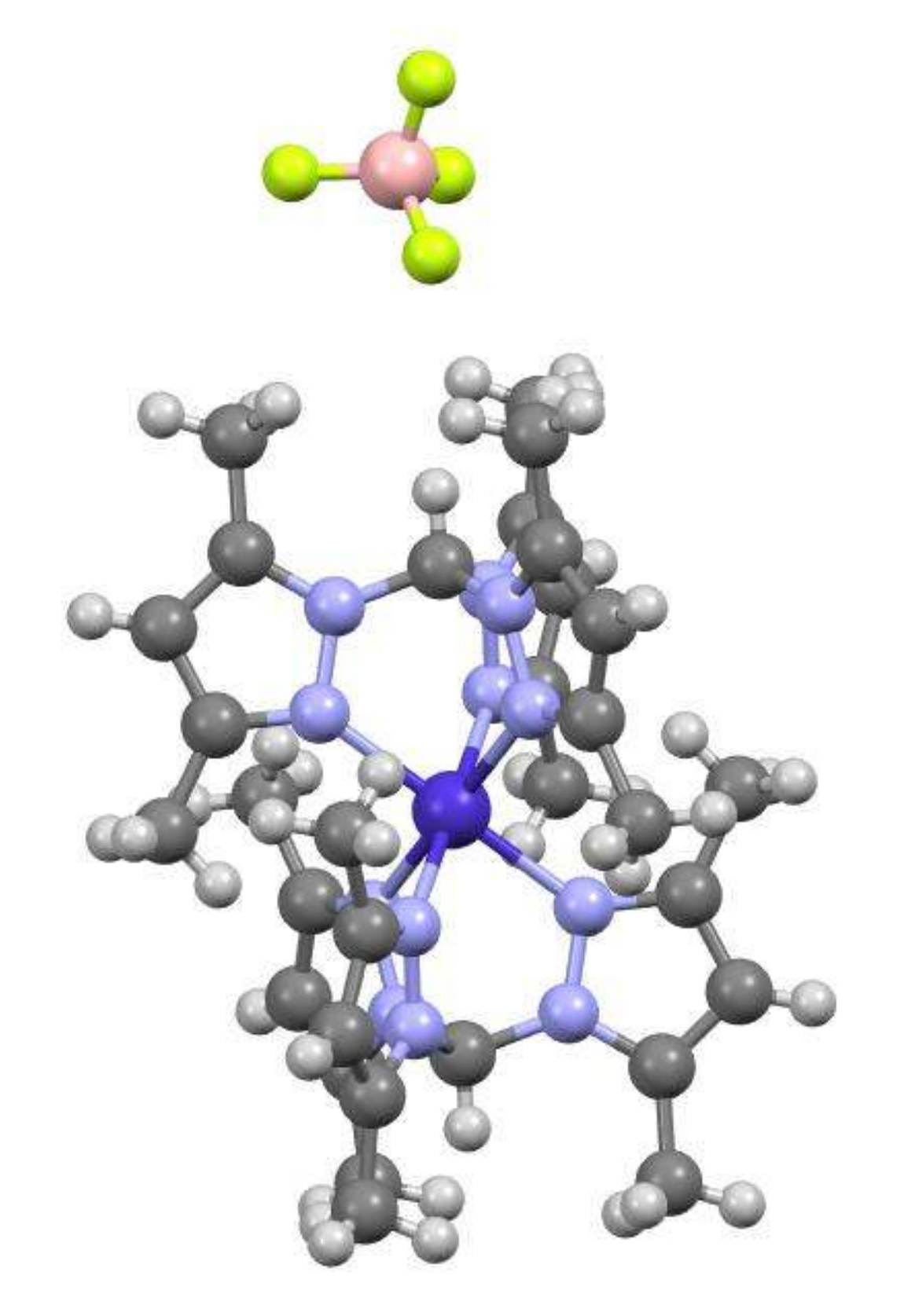

$\left[\mathrm{Co}\left(\mathrm{Tpm}^{3,5 \mathrm{me}}\right)_{2}\right]\left[\mathrm{BF}_{4}\right]$
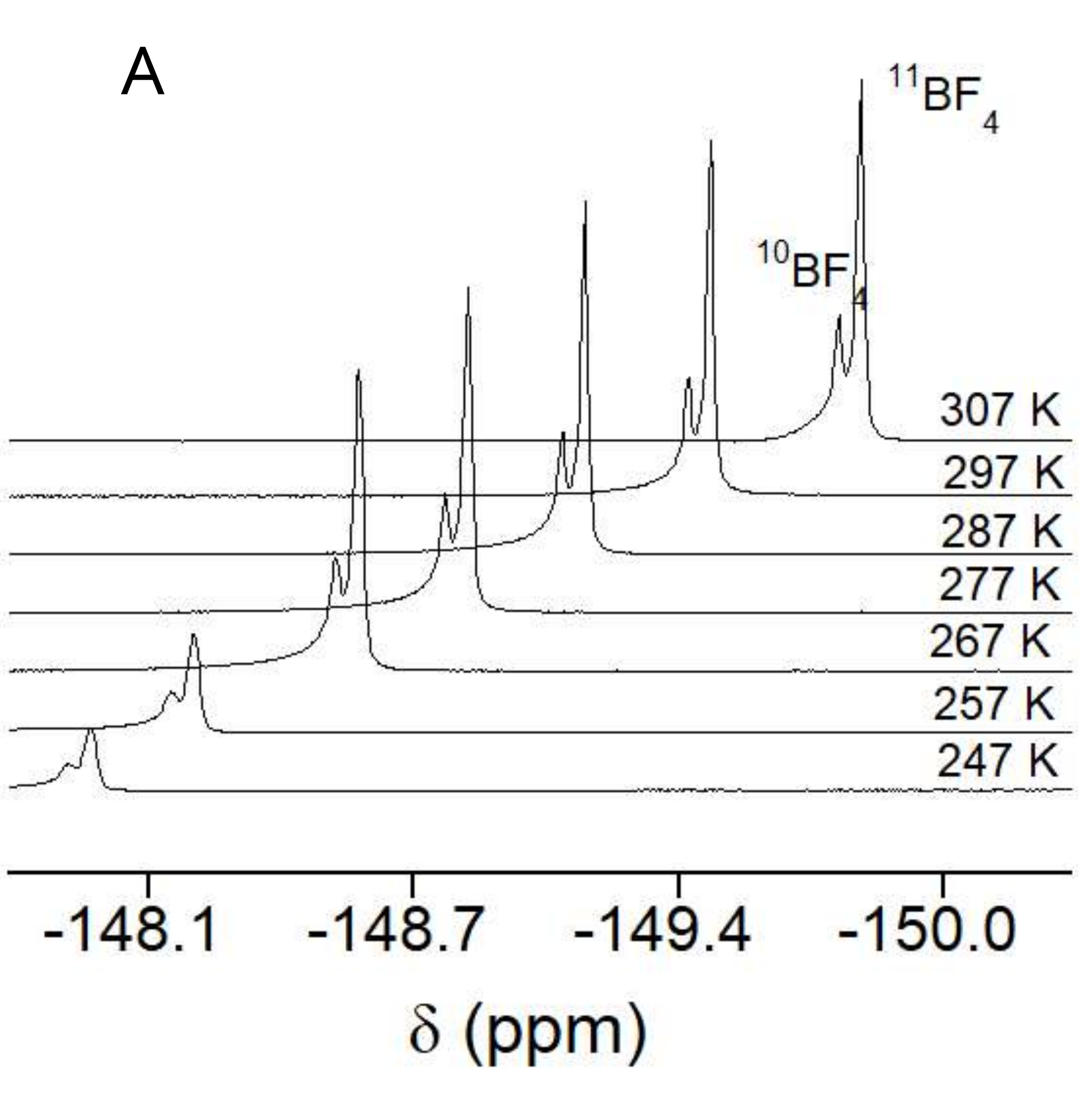

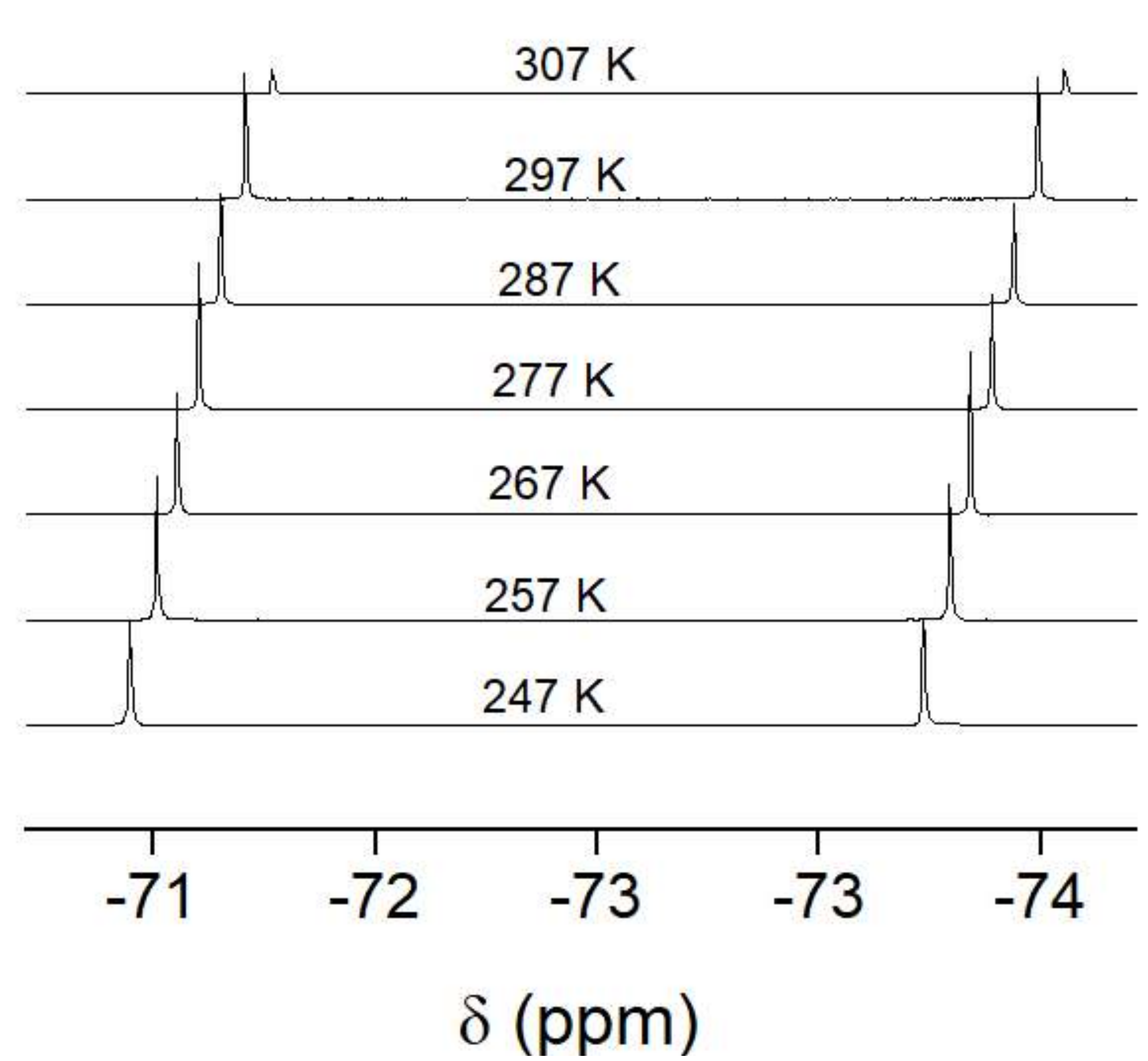

Table 1. Calculated and measured $\mathrm{Co}-{ }^{19} \mathrm{~F}$ distances of Tpm complexes in solution and crystal structure

\begin{tabular}{|c|c|c|}
\hline Complex & $\begin{array}{c}\text { Distance of } \\
\text { crystal structure } \\
\left(\mathrm{A}^{\circ}\right)\end{array}$ & $\begin{array}{c}\text { Distance of } \\
\text { crystal structure } \\
\left(\mathrm{A}^{\circ}\right)\end{array}$ \\
\hline$\left[\mathrm{Co}(\mathrm{Tpe})_{2}\right](\mathrm{BF} 4)_{2}$ & 6.172 & 8.973 \\
\hline$\left[\mathrm{Co}\left(\mathrm{Tpm}^{3,5 \mathrm{me}}\right)_{2}\right]\left(\mathrm{PF}_{6}\right)_{2}$ & $\begin{array}{l}6.005 \\
8.409\end{array}$ & 11.80 \\
\hline$\left[\mathrm{Co}\left(\mathrm{Tpm}^{3,5 \mathrm{me}}\right)_{2}\right]\left(\mathrm{BF}_{4}\right)_{2}$ & 8.220 & 9.454 \\
\hline$\left[\mathrm{Co}\left(\mathrm{Tpm}^{4 \mathrm{me}}\right)_{2}\right]\left(\mathrm{BF}_{4}\right)_{2}$ & 6.9005 & 8.034 \\
\hline
\end{tabular}

Figure 1. Variable-temperature ${ }^{19} \mathrm{~F}$ spectra of counter ion of $(\mathrm{A})\left[\mathrm{Co}(\mathrm{Tpe})_{2}\right]\left[\mathrm{BF}_{4}\right]$ and (B) $\left[\mathrm{Co}\left(\mathrm{Tpm}^{3,5 \mathrm{me}}\right)_{2}\right]\left[\mathrm{PF}_{6}\right]$

$$
r=\left[\frac{2}{15}\left(\frac{\mu_{0}}{4 \pi}\right) \frac{\gamma_{N} g_{e} \mu_{B} S(S+1)}{\mathrm{T}_{1 M(\text { dip })}^{-1}}\left(3 \cos ^{2} \vartheta-1\right)\left(\frac{7 \tau_{c}}{1+\varpi_{S}^{2} \tau_{c}^{2}}+\frac{3 \tau_{c}}{1+\varpi_{I}^{2} \tau_{c}^{2}}\right)\right]^{1 / 6}
$$

Equation 1. Modified Solomon-Bloembergen and Morgan equation

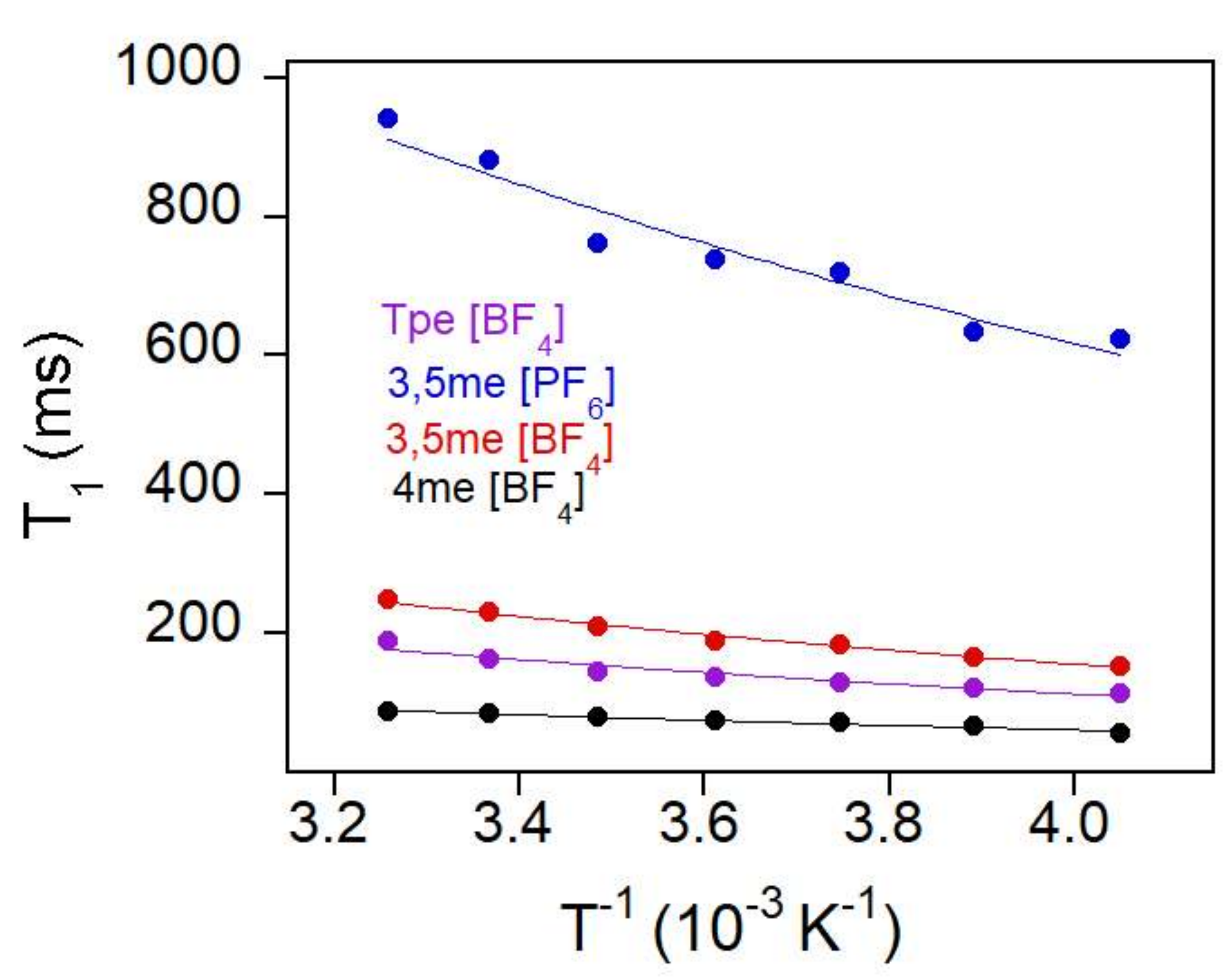

Figure 2. Temperature dependence of ${ }^{19} \mathrm{~F}$ spin-lattice relaxation time $\left(\mathrm{T}_{1}\right.$ at $300 \mathrm{MHz}$ for Tpe $\left[\mathrm{BF}_{4}\right], 4 \mathrm{me}$

$\left.\left.\left[\mathrm{BF}_{4}\right]\right)_{2}\right], 3,5 \mathrm{me}\left[\mathrm{PF}_{6}\right]$ and $3,5 \mathrm{me}\left[\mathrm{BF}_{4}\right]$

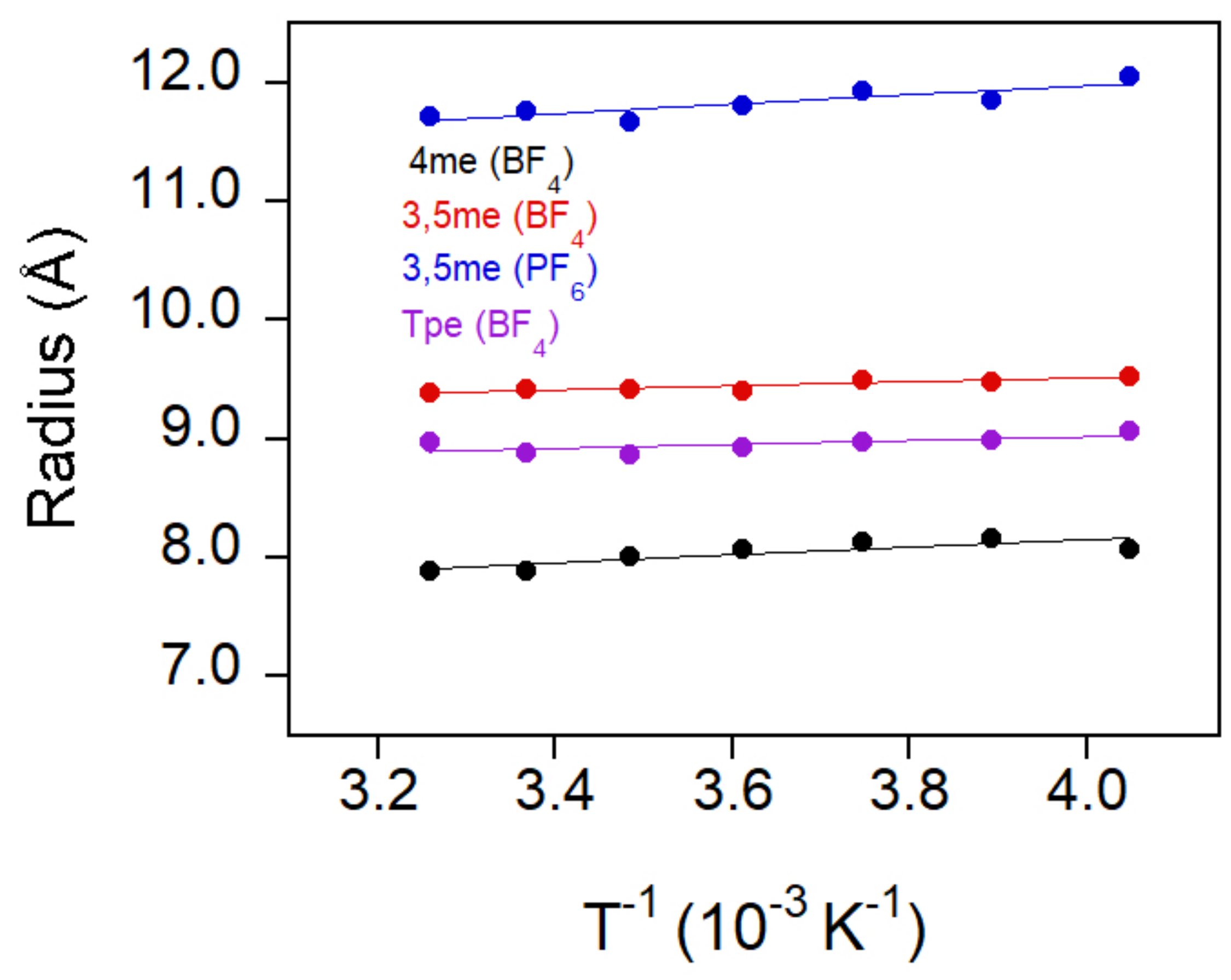
$\left[\mathrm{BF}_{4}\right]$

\section{Conclusion}

The $\mathrm{BF}_{4}^{-}$counterion nuclei relax faster and are more tightly associated with the cobalt metal center compared to $\mathrm{PF}_{6}{ }^{-}$ion in solution. NMR studies of substituted Tpm complexes suggest that counter ion nuclei in solution have a weak interaction with metal center compared to the crystal structure.

\section{Acknowledgement}

Special thanks to Dr. Ramelot for assistance with NMR and Dr. Rakovan for assistance of SC-XRD data analysis. The

Figure 4. calculated temperature dependence of distances between counter ion nuclei and metal center (BF4and $\mathrm{PF}_{6}$ ) in solution for Tpe [BF4] 4me $\left.[\mathrm{BF} 4])_{2}\right], 3,5 \mathrm{me}\left[\mathrm{PF}_{6}\right]$ and $3,5 \mathrm{me}$

\section{Literature cited}

Macchioni, A.; Magistrato, A.; Orabona, I.; Ruffo, F.; Rothlisberger, U. Zuccaccia, C. New J. Chem. 2003, 27 (3), 455-458.

2. Lin, C.-Y.; Ngendahimana, T.; Eaton, G. R.; Eaton, S. S.; Zadrozny, J. M. Chem. Sci. 2019, $10(2), 548-555$.

3. Marts, A. R.; Kaine, J. C.; Baum, R. R.; Clayton, V. L.; Bennett, J. R.; Cordonnier, L. J.; McCarrick, R.; Hasheminasab, A.; Crandall, L. A.; Ziegler, C. J. Inorg. Chem. 2016, 56 (1), 618-626 